\title{
Generalized Taylor Series Method for Solving Nonlinear Fractional Differential Equations with Modified Riemann-Liouville Derivative
}

\author{
Süleyman Öğrekçi \\ Sciences \& Arts Faculty, Amasya University, 05000 Amasya, Turkey \\ Correspondence should be addressed to Süleyman Öğrekçi; suleyman.ogrekci@amasya.edu.tr \\ Received 30 September 2014; Revised 8 January 2015; Accepted 18 March 2015 \\ Academic Editor: Claudio Dappiaggi \\ Copyright (C) 2015 Süleyman Öğrekçi. This is an open access article distributed under the Creative Commons Attribution License, \\ which permits unrestricted use, distribution, and reproduction in any medium, provided the original work is properly cited.

\begin{abstract}
We propose an efficient analytic method for solving nonlinear differential equations of fractional order. The fractional derivative is defined in the sense of modified Riemann-Liouville derivative. A new technique for calculating the generalized Taylor series coefficients (also known as "generalized differential transforms," GDTs) of nonlinear functions and a new approach of the generalized Taylor series method (GTSM) are presented. This new method offers a simple algorithm for computing GDTs of nonlinear functions and avoids massive computational work that usually arises in the standard method. Several illustrative examples are demonstrated to show effectiveness of the proposed method.
\end{abstract}

\section{Introduction}

Fractional differential equations are generalizations of classical differential equations of integer order and have recently proved to be valuable tools in the modeling of many phenomena in various fields of science and engineering. Apart from diverse areas of mathematics, fractional differential equations arise in rheology, viscoelasticity, chemical physics, electrical networks, fluid flows, control, and dynamical processes in self-similar and porous structures. There has appeared lots of work in which fractional derivatives are used for a better description of considered material properties; mathematical modelling based on enhanced rheological models naturally leads to differential equations of fractional order and to the necessity of the formulation of initial conditions to such equations. Several numerical methods for solving fractional differential equations have been introduced lately. The authors in [1] presented the predictor-corrector approach based on the Adam-Bashforth-Moulton type numerical method that has been successful in obtaining stable approximations for solving many fractional differential equations. Some of the semianalytic methods such as the Adomian decomposition method (ADM) $[2,3]$, homotopy analysis method (HAM) [4-6], homotopy perturbation method (HPM) [7, 8], variational iteration method (VIM) $[9,10]$, and generalized differential transform method (GDTM) [11-13] have been introduced to provide analytic or numeric approximations.

In this paper we focus on the generalized Taylor series method (GTSM), which is based on the generalized Taylor series. The fractional derivative is defined in the sense of the modified Riemann-Liouville derivative [14]. From the given fractional differential equation, the GTSM provides a simple recurrence relation of the generalized Taylor series coefficients of the solution. We obtain recurrence relations of complex nonlinear functions such as the exponential, logarithmic, and trigonometric functions. The paper is organized as follows. Section 2 introduces some preliminary results from the fractional calculus that we will use. The basic idea and some properties of GTSM are presented in Section 3. We present the recurrence relations of complex nonlinear functions in Section 4. In Section 5, numerical results of several examples are demonstrated by using new recurrence relations. Finally, we give a conclusion in Section 6. 


\section{Preliminary Results}

In [14], Jumarie proposed a definition for fractional derivative which is known as the modified Riemann-Liouville derivative in the literature. Since then, many authors have investigated various applications of the modified Riemann-Liouville derivative (e.g., see [15-17]) including various fractional calculus formulae, the fractional variational iteration method, and the fractional subequation method for solving fractional partial differential equations. The definition and some of the key properties of modified Riemann-Liouville derivative are

$$
\begin{aligned}
& D_{t}^{\alpha} f(t) \\
& = \begin{cases}\frac{1}{\Gamma(1-a)} \frac{d}{d t} & \\
\cdot \int_{0}^{t}(t-\xi)^{-\alpha}(f(\xi)-f(0)) d \xi, & 0<\alpha<1 \\
\left(f^{(n)}(t)\right)^{(\alpha-n)}, & 1 \leq n \leq \alpha<n+1,\end{cases} \\
& D_{t}^{\alpha} t^{\beta}=\frac{\Gamma(1+\beta)}{\Gamma(1+\beta-\alpha)} t^{\beta-\alpha}, \\
& D_{t}^{\alpha}(f(t) g(t))=g(t) D_{t}^{\alpha} f(t)+f(t) D_{t}^{\alpha} g(t) \\
& D_{t}^{\alpha} f(g(t))=f_{g}^{\prime}(g(t)) D_{t}^{\alpha} g(t)=D_{g}^{\alpha} f(g(t))\left(g^{\prime}(t)\right)^{\alpha},
\end{aligned}
$$

which do not hold for classical Riemann-Liouville and Caputo derivatives. Particularly chain rule (also known as "Faà di Bruno's formula" in fractional calculus) plays the key role in our method.

\section{Generalized Taylor Series Method}

The Taylor series method (TSM) (also known as differential transform method, DTM) that is based on the Taylor series has been successful in achieving accurate approximate solutions for the linear and nonlinear problems. In TSM, all coefficients of Taylor series of the solution can be determined by solving the recurrence equations induced from the given differential equation. The authors in [18] developed the fractional differential transform method (FDTM) which is based on the classical TSM and generalized with fractional derivative. The generalized Taylor formula is introduced in [11] and has been used to develop GDTM in [12]. The authors in [19] proposed a new algorithm for calculating the differential transforms of several nonlinear functions based on the chain rule. The purpose of this paper is to obtain efficient algorithms to calculate generalized Taylor series coefficients of complex nonlinear functions.

For the fractional differential operator $D^{\alpha}, m-1<\alpha \leq m$, in the sense of modified Riemann-Liouville definition, let us define $\left(D^{\alpha}\right)^{n}=D^{\alpha} \cdot D^{\alpha} \cdots D^{\alpha}(n$ times $)$.
Theorem 1 (generalized Taylor's formula [11]). Suppose that $\left(D_{t}^{\alpha}\right)^{k} f(t) \in C(0, b]$ for $k=0,1, \ldots, n+1$, where $0<\alpha \leq 1$; then one has

$$
f(t)=\sum_{i=0}^{n} \frac{t^{i \alpha}}{\Gamma(i \alpha+1)}\left(\left(D_{t}^{\alpha}\right)^{i} f\right)(0)+\frac{\left(\left(D_{t}^{\alpha}\right)^{n+1} f\right)(\eta)}{\Gamma((n+1) \alpha+1)} t^{(n+1) \alpha}
$$

for all $t \in(0, b]$ with $0 \leq \eta \leq t$.

For an analytic function $f(t)$, let us define the generalized differential transform (GDT) of the $k$ th derivative as follows:

$$
F(k)=\frac{1}{\Gamma(\alpha k+1)}\left[\left(D_{t}^{\alpha}\right)^{k} f(t)\right]_{t=0},
$$

where $0<\alpha \leq 1, k=0,1,2, \ldots$, and the generalized differential inverse transform of $F(k)$ is defined as follows:

$$
f(t)=\sum_{k=0}^{\infty} F(k) t^{\alpha k}
$$

Roughly speaking, coefficient of the $k$ th term in the generalized Taylor series of the function $f$ is called the GDT of the $k$ th derivative of $f$. In case of $\alpha=1$, the GDT reduces to the classical differential transform. Some of the fundamental properties of GDT are listed below.

Theorem 2. Suppose that $F(k), G(k)$, and $H(k)$ are the generalized differential transforms of the functions $f(t), g(t)$, and $h(t)$, respectively. Then the following properties are satisfied.

(1) If $f(t)=g(t) \pm h(t)$, then $F(k)=G(k) \pm H(k)$.

(2) If $f(t)=a g(t)$, where $a$ is a constant, then $F(k)=$ $a G(k)$.

(3) If $f(t)=g(t) h(t)$, then $F(k)=\sum_{l=0}^{k} G(l) H(k-l)$.

(4) If $f(t)=D_{t}^{\alpha} g(t)$, then $F(k)=(\Gamma(\alpha(k+1)+1) / \Gamma(\alpha k+$ 1)) $G(k+1)$.

The proof of these results for Caputo derivative can be found in [12] and clearly holds for modified RiemannLiouville derivative also.

\section{Calculating the Coefficients of Generalized Taylor Series for Complex Nonlinear Functions}

In this section we will introduce an efficient algorithm to calculate generalized Taylor series coefficients (GDTs) of several complex nonlinear functions. Since the chain rule holds for the modified Riemann-Liouville derivative, the algorithms for calculating the GDTs of typical nonlinear functions given in [19] can be totally adopted to nonlinear fractional differential equations with modified RiemannLiouville derivative. 
4.1. GDTs for the Exponential and Logarithmic Nonlinearity. Let us consider $f(y(t))=e^{a y(t)}$, where $a$ is a constant. From the definition of transform,

$$
F(0)=\left.e^{a y(t)}\right|_{t=0}=e^{a y(0)}=e^{a Y(0)} .
$$

Now taking fractional derivative of $f(y(t))=e^{a y(t)}$ with respect to variable $t$, we have

$$
D_{t}^{\alpha} f(y(t))=a e^{a y(t)} D_{t}^{\alpha} y(t)=a f(y(t)) D_{t}^{\alpha} y(t) .
$$

With the application of Theorem 2 to (6) to obtain the generalized Taylor series coefficients, we have

$$
\begin{aligned}
\frac{\Gamma(\alpha(k+1)+1)}{\Gamma(\alpha k+1)} F(k+1)= & a \sum_{l=0}^{k} \frac{\Gamma(\alpha(l+1)+1)}{\Gamma(\alpha l+1)} \\
& \cdot Y(l+1) F(k-l) .
\end{aligned}
$$

Now replacing $k+1$ with $k$ gives

$$
\begin{aligned}
F(k)= & a \frac{\Gamma(\alpha(k-1)+1)}{\Gamma(\alpha k+1)} \sum_{l=0}^{k-1} \frac{\Gamma(\alpha(l+1)+1)}{\Gamma(\alpha l+1)} \\
& \cdot Y(l+1) F(k-1-l)
\end{aligned}
$$

for $k \geq 1$. Combining (5) and (8), we obtain the recursive relationship for calculating GDTs of $f(y(t))=e^{a y(t)}$ :

$F(k)$

$$
= \begin{cases}e^{a Y(0)}, & k=0, \\ a \frac{\Gamma(\alpha(k-1)+1)}{\Gamma(\alpha k+1)} \sum_{l=0}^{k-1} \frac{\Gamma(\alpha(l+1)+1)}{\Gamma(\alpha l+1)} & \\ \cdot Y(l+1) F(k-1-l), & k \geq 1 .\end{cases}
$$

Now consider $f(y(t))=\ln (a+b y(t))$, where $a$ and $b$ are constants and $y$ is a continuous function satisfying $a+b y(t)>$ 0 for all $t \in \mathbb{R}$. By definition of transform, we have

$$
F(0)=\left.\ln (a+b y(t))\right|_{t=0}=\ln (a+b y(0))=\ln (a+b Y(0)) \text {. }
$$

By taking fractional derivative of $f(y(t))=\ln (a+b y(t))$ with respect to variable $t$, we have

$$
D_{t}^{\alpha} f(y(t))=\frac{b}{a+b y(t)} D_{t}^{\alpha} y(t)
$$

or equivalently

$$
a D_{t}^{\alpha} f(y(t))=b\left[D_{t}^{\alpha} y(t)-y(t) D_{t}^{\alpha} f(y(t))\right] .
$$

With the application of the generalized differential transform to (12) we have, from Theorem 2,

$$
\begin{aligned}
a F(k+1)=b\left[Y(k+1)-\frac{\Gamma(\alpha k+1)}{\Gamma(\alpha(k+1)+1)}\right. & \\
& \left.\cdot \sum_{l=0}^{k} \frac{\Gamma(\alpha(l+1)+1)}{\Gamma(\alpha l+1)} F(l+1) Y(k-l)\right] .
\end{aligned}
$$

Replacing $k+1$ with $k$ gives

$$
\begin{aligned}
a F(k)=b[ & Y(k)-\frac{\Gamma(\alpha(k-1)+1)}{\Gamma(\alpha k+1)} \\
& \left.\cdot \sum_{l=0}^{k-1} \frac{\Gamma(\alpha(l+1)+1)}{\Gamma(\alpha l+1)} F(l+1) Y(k-1-l)\right] .
\end{aligned}
$$

Substitute $k=1$ in (14) to get

$$
F(1)=\frac{b}{a+b Y(0)} Y(1)
$$

For $k \geq 2$, (14) can be rewritten as

$$
\begin{aligned}
F(k)= & \frac{b}{a+b Y(0)} \\
& \cdot\left[Y(k)-\frac{\Gamma(\alpha(k-1)+1)}{\Gamma(\alpha k+1)}\right. \\
& \left.\cdot \sum_{l=0}^{k-2} \frac{\Gamma(\alpha(l+1)+1)}{\Gamma(\alpha l+1)} F(l+1) Y(k-1-l)\right] .
\end{aligned}
$$

By combining (10), (15), and (16), we obtain the recursive relationship for calculating GDTM of $f(y(t))=\ln (a+b y(t))$ :

$$
\begin{aligned}
& F(k) \\
& =\left\{\begin{array}{cc}
\ln (a+b Y(0)), & k=0, \\
\frac{b}{a+b Y(0)} Y(1), & k=1, \\
\frac{b}{a+b Y(0)}\left[\begin{array}{l}
Y(k)-\frac{\Gamma(\alpha(k-1)+1)}{\Gamma(\alpha k+1)} \\
\sum_{l=0}^{k-2} \frac{\Gamma(\alpha(l+1)+1)}{\Gamma(\alpha l+1)}
\end{array}\right. & \\
\cdot F(l+1) Y(k-1-l)], & k \geq 2 .
\end{array}\right.
\end{aligned}
$$

4.2. GDTMs for the Trigonometric and Hyperbolic Nonlinearity. Now consider $f(y(t))=\sin (a y(t))$ and $g(y(t))=$ $\cos (a y(t))$, where $a$ and $b$ are constants. By definition of GDT,

$$
\begin{aligned}
& F(0)=\left.\sin (a y(t))\right|_{t=0}=\sin (a y(0))=\sin (a Y(0)), \\
& G(0)=\left.\cos (a y(t))\right|_{t=0}=\cos (a y(0))=\cos (a Y(0)) .
\end{aligned}
$$

Further, taking fractional derivative of (18), we have

$$
\begin{aligned}
& D_{t}^{\alpha} f(y(t))=a g(y(t)) D_{t}^{\alpha} y(t), \\
& D_{t}^{\alpha} g(y(t))=-a f(y(t)) D_{t}^{\alpha} y(t) .
\end{aligned}
$$


Similarly, applying GDT with the aid of Theorem 2, we have

$$
\begin{aligned}
& \frac{\Gamma(\alpha(k+1)+1)}{\Gamma(\alpha k+1)} F(k+1) \\
& \quad=a \sum_{l=0}^{k} \frac{\Gamma(\alpha(k-l+1)+1)}{\Gamma(\alpha(k-l)+1)} G(l) Y(k-l+1), \\
& \frac{\Gamma(\alpha(k+1)+1)}{\Gamma(\alpha k+1)} G(k+1) \\
& \quad=-a \sum_{l=0}^{k} \frac{\Gamma(\alpha(k-l+1)+1)}{\Gamma(\alpha(k-l)+1)} F(l) Y(k-l+1) .
\end{aligned}
$$

Replacing $k+1$ with $k$ gives

$$
\begin{aligned}
F(k)= & a \frac{\Gamma(\alpha(k-1)+1)}{\Gamma(\alpha k+1)} \\
& \cdot \sum_{l=0}^{k-1} \frac{\Gamma(\alpha(k-l)+1)}{\Gamma(\alpha(k-l-1)+1)} G(l) Y(k-l), \\
G(k)= & -a \frac{\Gamma(\alpha(k-1)+1)}{\Gamma(\alpha k+1)} \\
& \cdot \sum_{l=0}^{k-1} \frac{\Gamma(\alpha(k-l)+1)}{\Gamma(\alpha(k-l-1)+1)} F(l) Y(k-l) .
\end{aligned}
$$

Combine (18) and (21) to obtain recurrence relation:

$F(k)$

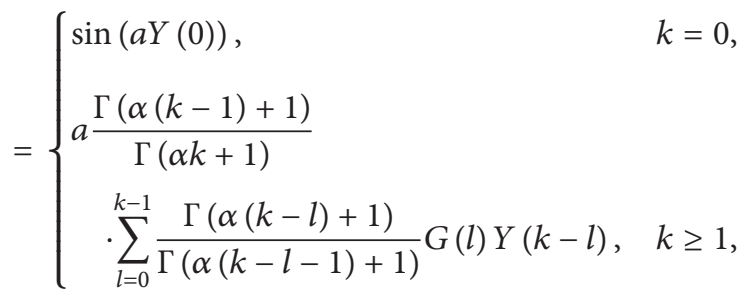

$G(k)$

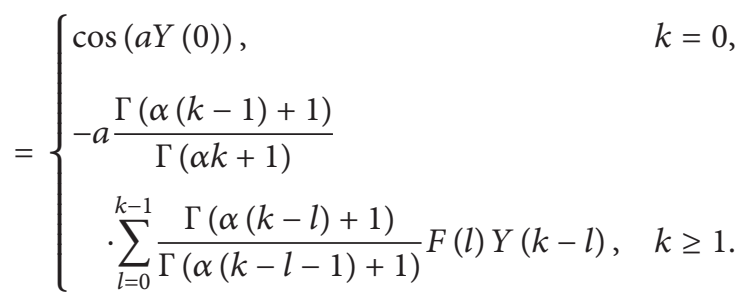

For the functions $f(y(t))=\sinh (a y(t))$ and $g(y(t))=$ $\cosh (a y(t))$, we immediately obtain the recursive relationships:

$F(k)$

$$
= \begin{cases}\sinh (a Y(0)), & k=0, \\ a \frac{\Gamma(\alpha(k-1)+1)}{\Gamma(\alpha k+1)} & \\ \cdot \sum_{l=0}^{k-1} \frac{\Gamma(\alpha(k-l)+1)}{\Gamma(\alpha(k-l-1)+1)} G(l) Y(k-l), & k \geq 1,\end{cases}
$$

$G(k)$

$$
= \begin{cases}\cosh (a Y(0)), & k=0, \\ a \frac{\Gamma(\alpha(k-1)+1)}{\Gamma(\alpha k+1)} & \\ \sum_{l=0}^{k-1} \frac{\Gamma(\alpha(k-l)+1)}{\Gamma(\alpha(k-l-1)+1)} F(l) Y(k-l), & k \geq 1 .\end{cases}
$$

4.3. Error Analysis. From Theorem 1, if $\left(D_{t}^{\alpha}\right)^{k} f(t) \in C(0, b]$ for $k=0,1, \ldots, n+1$, where $0<\alpha \leq 1$, then we have

$$
f(t) \cong S_{n}(t)=\sum_{i=0}^{n} \frac{t^{i \alpha}}{\Gamma(i \alpha+1)}\left(\left(D_{t}^{\alpha}\right)^{i} f\right)(0)
$$

for all $t \in(0, b]$. Furthermore, there is a value $\eta$ with $0 \leq \eta \leq t$ so that the error term $R_{n}^{\alpha}(t)$ has the form

$$
R_{n}^{\alpha}(t)=\frac{\left(\left(D_{t}^{\alpha}\right)^{n+1} f\right)(\eta)}{\Gamma((n+1) \alpha+1)} t^{(n+1) \alpha} .
$$

So, if $F(k)$ is GDT of the $k$ th derivative of the analytic function $f$, we have

$$
f(t) \cong S_{n}(t)=\sum_{k=0}^{n} F(k) t^{\alpha k},
$$

and the error term has the form

$$
R_{n}^{\alpha}(t)=F(n+1) \eta^{\alpha(n+1)}
$$

for some $\eta \in[0, t]$. Absolute value of the error done with approximation can be calculated by maximizing the right hand side of following inequality:

$$
\left|R_{n}^{\alpha}(t)\right|=|F(n+1)| \eta^{\alpha(n+1)}
$$

for $\eta \in[0, t]$. The accuracy of $P_{k}^{\alpha}(t)$ increases when we choose large $k$ and decreases as the value of $t$ moves away from the center 0 . Hence, we must choose $k$ large enough so that the error does not exceed a specified bound.

\section{Numerical Illustrations}

Example 1. Consider the following nonlinear fractional differential equation:

$$
D_{t}^{\alpha} y(t)+e^{y(t)}=0
$$


TABLE 1: $F(k)$ and $Y(k)$ values in Example 1 for $\alpha=0.9, t=0.05$, and $k \leq 10$.

\begin{tabular}{ccc}
\hline$k$ & $F(k)$ & $Y(k)$ \\
\hline 0 & 1 & 0 \\
1 & -1.039754 & -1.039754 \\
2 & 1.241336 & 0.644852 \\
3 & -1.146326 & -0.177535 \\
4 & 0.955850 & 0.020540 \\
5 & -0.732814 & -0.001364 \\
6 & 0.521868 & 0.000058 \\
7 & -0.350469 & $-1.7 \times 10^{-6}$ \\
8 & 0.223641 & $3.64 \times 10^{-8}$ \\
9 & -0.136464 & $-5.92 \times 10^{-10}$ \\
10 & 0.080040 & $7.52 \times 10^{-12}$ \\
\hline
\end{tabular}

TABLE 2: Approximate solutions with the error bound $\left|R_{5}^{0.9}(t)\right| \leq$ $5.480973 \times 10^{-8}$ in Example 1 obtained by GTSM for $\alpha=0.9$.

\begin{tabular}{lc}
\hline$t$ & $y(t) \approx S_{n}(t): n=5$ \\
\hline 0.01 & -0.016317 \\
0.02 & -0.030191 \\
0.03 & -0.043136 \\
0.04 & -0.055448 \\
0.05 & -0.067265 \\
0.06 & -0.078667 \\
0.07 & -0.089710 \\
0.08 & -0.100432 \\
0.09 & -0.110863 \\
0.1 & -0.121026 \\
\hline
\end{tabular}

for $t>0$ and $0<\alpha<1$, subject to initial condition $y(0)=0$. Applying GTSM to (30) with the aid of Theorem 2, we have the following recurrence relation:

$$
\frac{\Gamma(\alpha(k+1)+1)}{\Gamma(\alpha k+1)} Y(k+1)+F(k)=0, \quad k \geq 0,
$$

where $F(k)$ is the GDT of $e^{y(t)}$ and is given by (9) as

$$
\begin{aligned}
F(k)= & a \frac{\Gamma(\alpha(k-1)+1)}{\Gamma(\alpha k+1)} \sum_{l=0}^{k-1} \frac{\Gamma(\alpha(l+1)+1)}{\Gamma(\alpha l+1)} \\
& \cdot Y(l+1) F(k-1-l)
\end{aligned}
$$

for $k \geq 1$. From the initial condition $y(0)=0$, we have $Y(0)=$ 0 and $F(0)=e^{Y(0)}=1$.

Solving the recurrence relations (31) and (32) together gives all GDTs. Table 1 shows the values of $F(k)$ and $Y(k)$ for $\alpha=0.9, t=0.05$, and $k \leq 10$. We set the number of iteration $n=5$ which gives the approximation $y(t) \approx$ $S_{n}(t)=\sum_{k=0}^{n} Y(k) t^{\alpha k}$ and the absolute error bound $\left|R_{5}^{0.9}(t)\right|=$ $\left[|Y(6)| \eta^{6}\right]_{\eta \in[0,0.05]} \leq 5.480973 \times 10^{-8}$. We see that the speed of convergence of $Y(k)$ is sufficient to obtain accurate approximations even in 5 iterations. In Table 2 , we summarize these approximations of solution for $\alpha=0.9$ and $t=$ $0.01, \ldots, 0.1$.

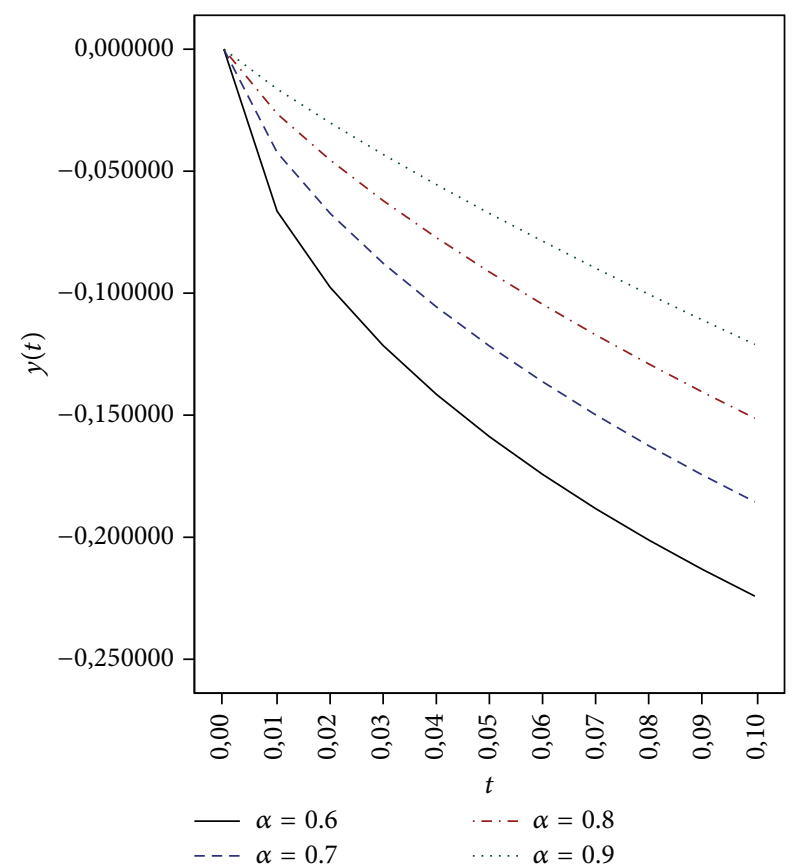

FIGURE 1: Approximate solutions obtained by GTSM in Example 1 for different order of derivatives.

Approximations of the solution $y(t)$ for different order of fractional derivatives are given in Figure 1. We set iteration number $n=5$ and summarize approximate solutions for $\alpha=$ $0,6, \ldots, 0.9$.

Example 2. Consider the nonlinear fractional differential equation

$$
D_{t}^{\alpha} y(t)=y(t)+y(t) \ln (y(t))
$$

for $t>0$ and $0<\alpha<1$, with the initial data $y(0)=1$. Applying GTSM to (33), we have the following recurrence relation:

$$
\frac{\Gamma(\alpha(k+1)+1)}{\Gamma(\alpha k+1)} Y(k+1)=Y(k)+\sum_{l=0}^{k} Y(l) F(k-l),
$$

where $F(k)$ is the GDT of $\ln (y(t))$ and is given by (17) as

$$
F(k)
$$

$$
= \begin{cases}\ln (Y(0)), & k=0, \\ \frac{Y(1)}{Y(0)} & k=1, \\ \frac{1}{Y(0)}\left[Y(k)-\frac{\Gamma(\alpha(k-1)+1)}{\Gamma(\alpha k+1)}\right. & \\ \cdot \sum_{l=0}^{k-2} \frac{\Gamma(\alpha(l+1)+1)}{\Gamma(\alpha l+1)} & \\ \cdot F(l+1) Y(k-1-l)], & k \geq 2 .\end{cases}
$$


TABLE 3: $F(k)$ and $Y(k)$ values in Example 2 for $\alpha=0.9, t=0.05$, and $k \leq 10$.

\begin{tabular}{ccc}
\hline$k$ & $F(k)$ & $Y(k)$ \\
\hline 0 & 0 & 1 \\
1 & 1.039754 & 1.039754 \\
2 & 0.596484 & 1.192968 \\
3 & 0.239770 & 1.153880 \\
4 & 0.074731 & 1.014274 \\
5 & 0.019104 & 0.830763 \\
6 & 0.004152 & 0.642560 \\
7 & 0.000786 & 0.473775 \\
8 & 0.0000132 & 0.335368 \\
9 & 0.000020 & 0.229136 \\
10 & $2.7 \times 10^{-7}$ & 0.151740 \\
\hline
\end{tabular}

From the initial data $y(0)=1$, we have $Y(0)=1$ and $F(0)=$ $\ln (Y(0))=0$. By using this in (34), we have

$$
Y(1)=\frac{1}{\Gamma(\alpha+1)}(Y(0)+Y(0) Y(1))=\frac{1}{\Gamma(\alpha+1)} .
$$

And by using this in (35) we obtain

$$
F(1)=\frac{Y(1)}{Y(0)}=\frac{1}{\Gamma(\alpha+1)}
$$

So we have

$$
\begin{aligned}
& F(k) \\
& = \begin{cases}0, & k=0, \\
\frac{1}{\Gamma(\alpha+1)}, & k=1, \\
Y(k)-\frac{\Gamma(\alpha(k-1)+1)}{\Gamma(\alpha k+1)} \sum_{l=0}^{k-2} \frac{\Gamma(\alpha(l+1)+1)}{\Gamma(\alpha l+1)} & \\
\cdot F(l+1) Y(k-1-l), & k \geq 2 .\end{cases}
\end{aligned}
$$

Solving the recurrence relations (34) and (38) together gives all GDTs. Table 3 shows the values of $F(k)$ and $Y(k)$ for $\alpha=0.9, t=0.05$, and $k \leq 10$. From Table 4, we see that the speed of convergence of $Y(k)$ is sufficient to obtain accurate approximations with 10 iterations. We set the number of iteration $n=10$ which gives the approximation $y(t) \approx S_{n}(t)=\sum_{k=0}^{n} Y(k) t^{\alpha k}$ and the error bound $\left|R_{10}^{0.9}(t)\right|=$ $\left[|Y(11)| \eta^{11}\right]_{\eta \in[0,0.05]} \leq|Y(11)| 0.05^{(11)}$. We summarize the approximations of solution for $\alpha=0.9$ and $t=0.01, \ldots, 0.1$ in Figure 2 and Table 5.

Example 3. Consider the fractional differential equation

$$
D_{t}^{\alpha} y(t)+\sin (y(t))=0
$$

TABLE 4: $S_{n}(t)$ and $\left|R_{n}^{\alpha}(t)\right|$ values in Example 2 for $\alpha=0.9, t=0.05$.

\begin{tabular}{llc}
\hline$n$ & $y(t) \approx S_{n}(t)$ & $\left|R_{n}^{\alpha}(t)\right|$ \\
\hline 5 & 1.075952287 & $6.058317 \times 10^{-8}$ \\
10 & 1.075952351 & $1.287679 \times 10^{-14}$ \\
50 & 1.075952351 & $1.281773 \times 10^{-73}$ \\
100 & 1.075952351 & $1.378753 \times 10^{-152}$ \\
500 & 1.075952351 & $2.864704 \times 10^{-783}$ \\
1000 & 1.075952351 & $1.358354 \times 10^{-1554}$ \\
\hline
\end{tabular}

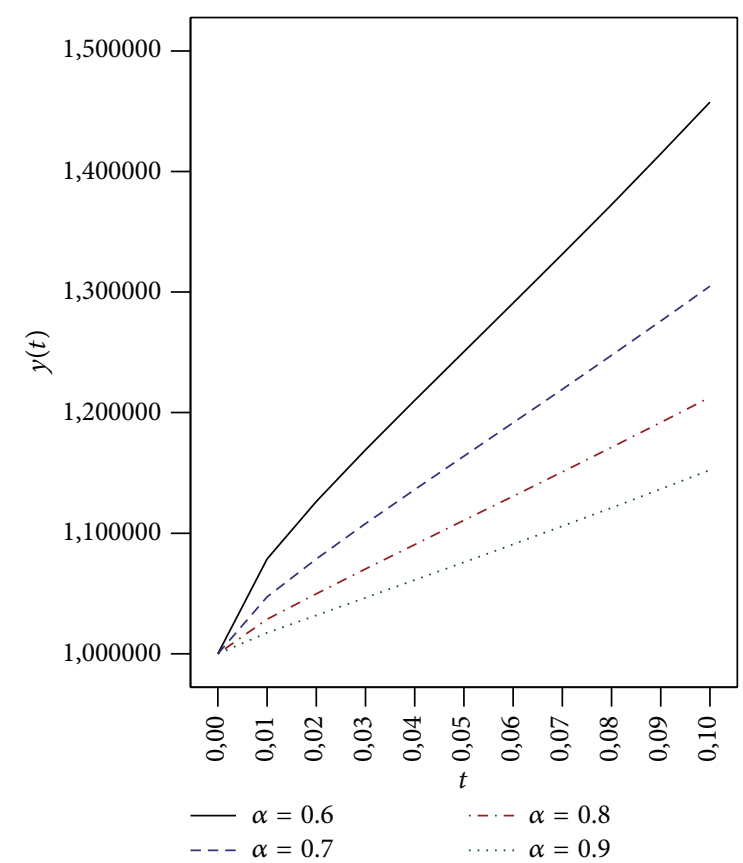

FIGURE 2: Approximate solutions obtained by GTSM in Example 2 for different order of derivatives.

for $t>0$ and $0<\alpha<1$, with the initial data $y(0)=3 \pi / 4$. Applying GTSM to (39), we have the following recurrence relation:

$$
\frac{\Gamma(\alpha(k+1)+1)}{\Gamma(\alpha k+1)} Y(k+1)+F(k)=0,
$$

where $F(k)$ is the GDT of $\sin (y(t))$ and is given by (22) as

$$
F(k)=\frac{\Gamma(\alpha(k-1)+1)}{\Gamma(\alpha k+1)} \sum_{l=0}^{k-1} \frac{\Gamma(\alpha(k-l)+1)}{\Gamma(\alpha(k-l-1)+1)} G(l) Y(k-l)
$$

and $G(k)$ is the GDT of $\cos (y(t))$ which is given by (23) as

$$
\begin{aligned}
G(k)= & -\frac{\Gamma(\alpha(k-1)+1)}{\Gamma(\alpha k+1)} \sum_{l=0}^{k-1} \frac{\Gamma(\alpha(k-l)+1)}{\Gamma(\alpha(k-l-1)+1)} \\
& \cdot F(l) Y(k-l)
\end{aligned}
$$

for $k \geq 1$.

From the initial data $y(0)=3 \pi / 4$, we have $Y(0)=3 \pi / 4$, $F(0)=\sin (3 \pi / 4)=\sqrt{2} / 2$, and $G(0)=\cos (3 \pi / 4)=-\sqrt{2} / 2$. 
TABLE 5: Approximate solutions obtained by GTSM in Example 2 for different order of derivatives.

\begin{tabular}{lcccc}
\hline$t$ & $y(t) \approx S_{n}(t)$ for $\alpha=0.06$ & $y(t) \approx S_{n}(t)$ for $\alpha=0.07$ & $y(t) \approx S_{n}(t)$ for $\alpha=0.08$ & $y(t) \approx S_{n}(t)$ for $\alpha=0.09$ \\
\hline 0.01 & 1.078558 & 1.047288 & 1.028575 & 1.017330 \\
0.02 & 1.126255 & 1.078509 & 1.049768 & 1.031825 \\
0.03 & 1.169182 & 1.107859 & 1.070435 & 1.046551 \\
0.04 & 1.210235 & 1.136106 & 1.090613 & 1.061220 \\
0.05 & 1.250579 & 1.163895 & 1.110622 & 1.075952 \\
0.06 & 1.290840 & 1.191577 & 1.130639 & 1.090816 \\
0.07 & 1.331417 & 1.219377 & 1.150782 & 1.105859 \\
0.08 & 1.372593 & 1.247450 & 1.171131 & 1.121118 \\
0.09 & 1.414586 & 1.275919 & 1.191753 & 1.136620 \\
0.1 & 1.457579 & 1.304879 & 1.212697 & 1.152390 \\
\hline
\end{tabular}

TABLE 6: $F(k)$ and $Y(k)$ values in Example 3 for $\alpha=0.9, t=0.05$, and $k \leq 10$.

\begin{tabular}{cccc}
\hline$k$ & $F(k)$ & $G(k)$ & $Y(k)$ \\
\hline 0 & 0.707106 & -0.707106 & 2.356194 \\
1 & 0.519877 & 0.519877 & -0.735217 \\
2 & $1.14 \times 10^{-10}$ & 0.421777 & -0.298242 \\
3 & -0.228527 & 0.108642 & $4.61 \times 10^{-11}$ \\
4 & -0.142652 & -0.100730 & 0.071226 \\
5 & 0.008355 & -0.112319 & 0.036468 \\
6 & 0.066994 & -0.029668 & -0.001815 \\
7 & 0.039284 & 0.029484 & -0.012690 \\
8 & -0.003954 & 0.032013 & -0.006604 \\
9 & -0.019730 & 0.007836 & 0.000598 \\
10 & -0.011001 & -0.008954 & 0.002717 \\
\hline
\end{tabular}

TABLE 7: $S_{n}(t)$ and $\left|R_{n}^{\alpha}(t)\right|$ values in Example 3 for $\alpha=0.9, t=0.05$.

\begin{tabular}{lcc}
\hline$n$ & $y(t) \approx S_{n}(t)$ & $\left|R_{n}^{\alpha}(t)\right|$ \\
\hline 0 & 2.356194490 & 0.049600 \\
1 & 2.306593692 & 0.001357 \\
2 & 2.305236270 & $1.416166 \times 10^{-14}$ \\
3 & 2.305236270 & $1.475491 \times 10^{-6}$ \\
4 & 2.305237745 & $5.096609 \times 10^{-8}$ \\
5 & 2.305237796 & $1.712146 \times 10^{-10}$ \\
10 & 2.305237796 & $1.833138 \times 10^{-16}$ \\
50 & 2.305237796 & $5.118854 \times 10^{-71}$ \\
100 & 2.305237796 & $7.834481 \times 10^{-139}$ \\
\hline
\end{tabular}

Solving the recurrence relations (40), (41), and (42) together gives all GDTs. Table 5 shows the values of $F(k)$ and $Y(k)$ for $\alpha=0.9, t=0.05$, and $k \leq 10$. Table 6 seems a bit complicated and does not give hint about speed of convergence of approximations; however, we see from Table 7 that the speed of convergence of $S_{n}(t)$ is sufficient to obtain accurate approximations even with 5 steps of iteration. We set the number of iteration $n=5$ which gives the approximation $y(t) \approx S_{n}(t)=\sum_{k=0}^{n} Y(k) t^{\alpha k}$ and the error bound $\left|R_{5}^{0.9}(t)\right|=$ $\left[|Y(6)| \eta^{6}\right]_{\eta \in[0,0.05]} \leq|Y(6)| 0.05^{6} \leq 1.712146 \times 10^{-10}$. Approximations of the solution $y(t)$ for different order of

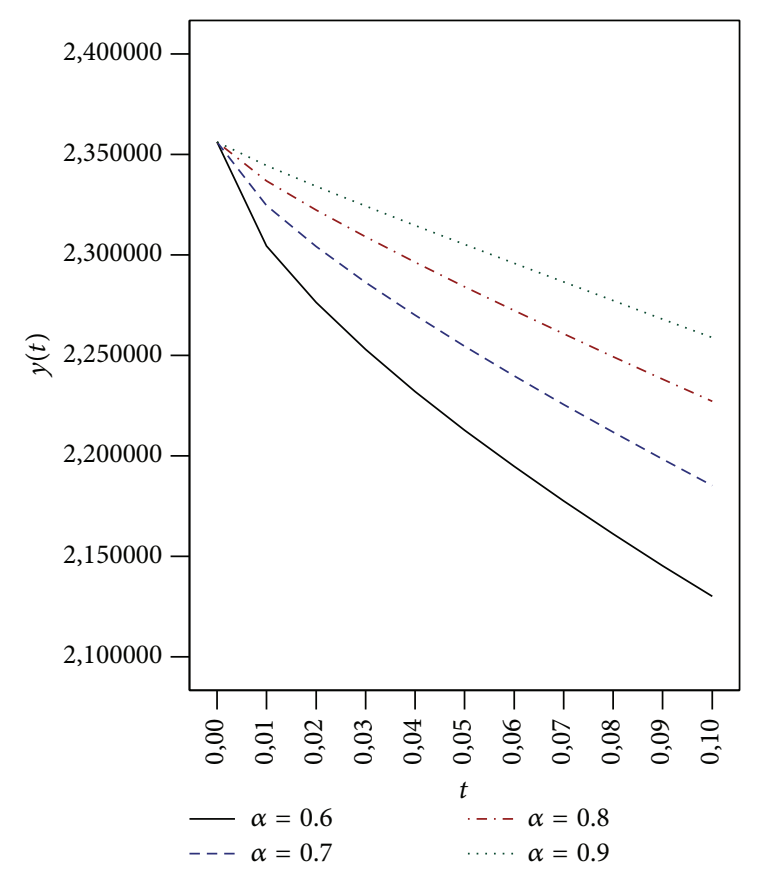

FIGURE 3: Approximate solutions obtained by GTSM in Example 3 for different order of derivatives.

fractional derivatives are given in Table 8 and Figure 3 . We set iteration number $n=5$ and summarize approximate solutions for $\alpha=0,6, \ldots, 0.9$.

Example 4. Consider the nonlinear fractional differential equation

$$
D_{t}^{\alpha} y(t)-\sinh (y(t))-\cos (y(t))=0
$$

for $t>0$ and $0<\alpha<1$, with the initial data $y(0)=0$. Applying GTSM to (43), we have the following recurrence relations:

$$
Y(k+1)=\frac{\Gamma(\alpha k+1)}{\Gamma(\alpha(k+1)+1)}\left[F_{1}(k)+G_{1}(k)\right],
$$


TABLE 8: Approximate solutions obtained by GTSM in Example 3 for different order of derivatives.

\begin{tabular}{lcccc}
\hline$t$ & $y(t) \approx S_{n}(t)$ for $\alpha=0.06$ & $y(t) \approx S_{n}(t)$ for $\alpha=0.07$ & $y(t) \approx S_{n}(t)$ for $\alpha=0.08$ & $y(t) \approx S_{n}(t)$ for $\alpha=0.09$ \\
\hline 0.01 & 2.304460 & 2.324576 & 2.336903 & 2.344467 \\
0.02 & 2.276386 & 2.304185 & 2.322322 & 2.334189 \\
0.03 & 2.252983 & 2.286388 & 2.308990 & 2.324333 \\
0.04 & 2.232084 & 2.270016 & 2.296360 & 2.341710 \\
0.05 & 2.212826 & 2.254587 & 2.284196 & 2.305237 \\
0.06 & 2.194756 & 2.239842 & 2.272369 & 2.295867 \\
0.07 & 2.177605 & 2.225624 & 2.260779 & 2.286568 \\
0.08 & 2.161196 & 2.211829 & 2.249432 & 2.277322 \\
0.09 & 2.145406 & 2.198384 & 2.238231 & 2.268112 \\
0.1 & 2.130145 & 2.185236 & 2.227165 & 2.258928 \\
\hline
\end{tabular}

TABLE 9: $F_{1}(k), F_{2}(k), G_{1}(k), G_{2}(k)$, and $Y(k)$ values in Example 4 for $\alpha=0.9, t=0.05$, and $k \leq 10$.

\begin{tabular}{lccccc}
\hline$k$ & $F_{1}(k)$ & $F_{2}(k)$ & $G_{1}(k)$ & $G_{2}(k)$ & 0 \\
\hline 0 & 0 & 1 & 1 & -1.039754 & 0 \\
1 & -1.039754 & 0 & 0 & 0.569484 & -1.039754 \\
2 & 0.569484 & 0.569484 & 0.569484 & 0.239770 & 0.569484 \\
3 & -0.239770 & -0.674339 & 0.674339 & -0.538923 & -0.135445 \\
4 & 0.268032 & -0.268032 & -0.118570 & 0.171348 & -0.038209 \\
5 & -0.285977 & 0.100162 & -0.317020 & 0.229499 & 0.131055 \\
6 & 0.138927 & -0.198550 & 0.181588 & -0.196168 & 0.060712 \\
7 & -0.044360 & 0.134687 & 0.165109 & -0.085070 & -0.020301 \\
8 & 0.088423 & -0.039427 & -0.222951 & 0.194067 & 0.020357 \\
9 & -0.126689 & 0.031339 & 0.005124 & -0.049923 & 0.016741 \\
10 & 0.077910 & 0.140686 & & \\
\hline
\end{tabular}

where $F_{1}(k)$ and $G_{1}(k)$ are the GDTs of $\sinh (y(t))$ and $\cos (y(t))$, which are given by

$$
\begin{aligned}
F_{1}(k)= & \frac{\Gamma(\alpha(k-1)+1)}{\Gamma(\alpha k+1)} \sum_{l=0}^{k-1} \frac{\Gamma(\alpha(k-l)+1)}{\Gamma(\alpha(k-l-1)+1)} \\
& \cdot F_{2}(l) Y(k-l), \\
F_{2}(k)= & \frac{\Gamma(\alpha(k-1)+1)}{\Gamma(\alpha k+1)} \sum_{l=0}^{k-1} \frac{\Gamma(\alpha(k-l)+1)}{\Gamma(\alpha(k-l-1)+1)} \\
& \cdot F_{1}(l) Y(k-l), \\
G_{1}(k)= & -\frac{\Gamma(\alpha(k-1)+1)}{\Gamma(\alpha k+1)} \sum_{l=0}^{k-1} \frac{\Gamma(\alpha(k-l)+1)}{\Gamma(\alpha-l-1)+1)} \\
& \cdot G_{2}(l) Y(k-l), \\
G_{2}(k)= & \frac{\Gamma(\alpha(k-1)+1)}{\Gamma(\alpha k+1)} \sum_{l=0}^{k-1} \frac{\Gamma(\alpha(k-l)+1)}{\Gamma(\alpha-l-1)+1)} \\
& \cdot G_{1}(l) Y(k-l)
\end{aligned}
$$

TABLE 10: $S_{n}(t)$ values in Example 4 for $\alpha=0.9, t=0.05$.

\begin{tabular}{lcc}
\hline$n$ & $y(t) \approx S_{n}(t)$ & $\left|R_{n}^{\alpha}(t)\right|$ \\
\hline 0 & 0 & 0 \\
1 & -0.07014612096 & 0.002714 \\
2 & -0.06743127723 & $7.405723 \times 10^{-14}$ \\
3 & -0.06743127723 & $2.805799 \times 10^{-6}$ \\
5 & -0.06743413643 & $1.235645 \times 10^{-8}$ \\
10 & -0.06743412447 & $3.642307 \times 10^{-15}$ \\
50 & -0.06743412447 & $4.457207 \times 10^{-66}$ \\
100 & -0.06743412447 & $2.306171 \times 10^{-129}$ \\
1000 & -0.06743412447 & $7.405826 \times 10^{-1260}$ \\
\hline
\end{tabular}

for $k \geq 1$. From the initial data $y(0)=0$, we have $Y(0)=0$, $F_{1}(0)=\sinh 0=0, F_{2}(0)=\cosh (0)=1, G_{1}(0)=\cos 0=1$, and $G_{2}(0)=\sin (0)=0$. Solving the recurrence relations (44), (45) together gives all GDTs.

Table 9 gives the values of $F_{1}(k), F_{2}(k), G_{1}(k), G_{2}(k)$, and $Y(k)$ for $\alpha=0.9, t=0.05$, and $k \leq 10$. We see from Table 10 that the speed of convergence of $S_{n}(t)$ is sufficient to obtain accurate approximations with 10 steps of iteration. Approximations of the solution $y(t)$ for different order of fractional derivatives are given in Figure 4. We set iteration number $n=10$ and summarize approximate solutions for $\alpha=0,6, \ldots, 0.9$. The numerical values are also given in Table 11. 
TABLE 11: Approximate solutions obtained by GTSM in Example 4 for different order of derivatives.

\begin{tabular}{lcccc}
\hline$t$ & $y(t) \approx S_{n}(t)$ for $\alpha=0.06$ & $y(t) \approx S_{n}(t)$ for $\alpha=0.07$ & $y(t) \approx S_{n}(t)$ for $\alpha=0.08$ & $y(t) \approx S_{n}(t)$ for $\alpha=0.09$ \\
\hline 0.01 & -0.067009 & -0.042538 & -0.026528 & -0.016329 \\
0.02 & -0.098771 & -0.067813 & -0.045619 & -0.030229 \\
0.03 & -0.123115 & -0.088613 & -0.062391 & -0.043211 \\
0.04 & -0.143374 & -0.106779 & -0.077707 & -0.055567 \\
0.05 & -0.160910 & -0.123103 & -0.091953 & -0.067434 \\
0.06 & -0.176454 & -0.138023 & -0.105349 & -0.078890 \\
0.07 & -0.190450 & -0.151813 & -0.118037 & -0.089990 \\
0.08 & -0.203197 & -0.164664 & -0.130118 & -0.100769 \\
0.09 & -0.214909 & -0.176712 & -0.141664 & -0.111258 \\
0.1 & -0.225742 & -0.186063 & -0.152733 & -0.121478 \\
\hline
\end{tabular}

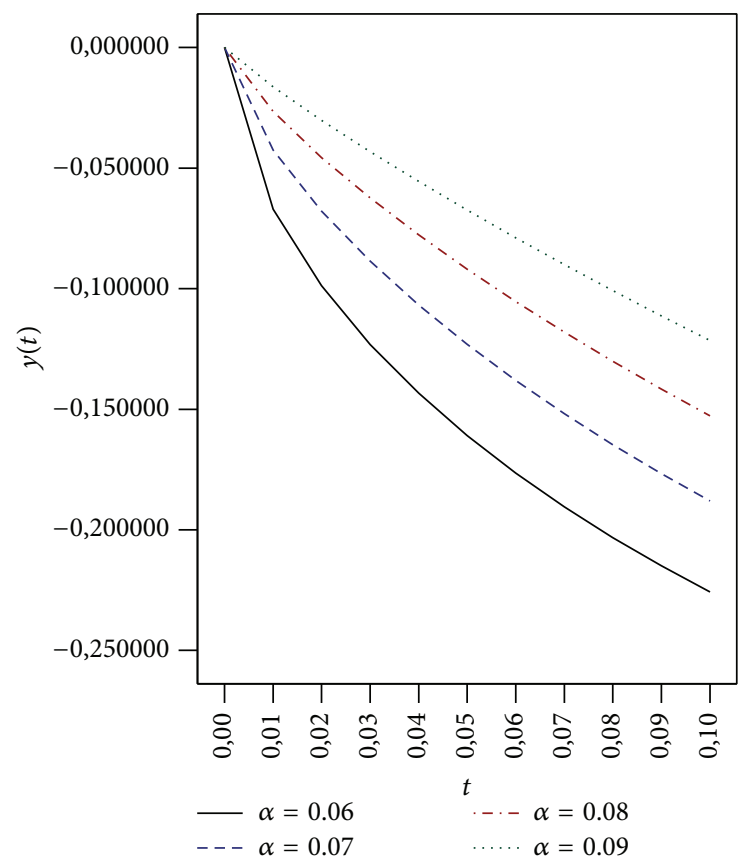

FIGURE 4: Approximate solutions obtained by GTSM in Example 4 for different order of derivatives.

\section{Conclusion}

In this study we proposed a generalized Taylor series method (GTSM) based on the generalized Taylor formula for solving nonlinear differential equations of fractional order. We consider the fractional differential equations with modified Riemann-Liouville derivative. In GTSM, it is key to obtain recursive relationships from the given differential equation. However, the recurrence relations for the complex nonlinear functions for fractional differential equations with modified Riemann-Liouville derivative have not been derived before. The GTSM with new recurrence relations given in this study offers a simple algorithm to compute the generalized Taylor series coefficients. Thus, it is worthwhile to mention that the GTSM is a straightforward, promising, and powerful method for solving nonlinear fractional differential equations.

\section{Conflict of Interests}

The author declares that there is no conflict of interests regarding the publication of this paper.

\section{References}

[1] K. Diethelm, N. J. Ford, and A. D. Freed, "A predictor-corrector approach for the numerical solution of fractional differential equations," Nonlinear Dynamics, vol. 29, no. 1-4, pp. 3-22, 2002.

[2] S. S. Ray and R. K. Bera, "An approximate solution of a nonlinear fractional differential equation by Adomian decomposition method," Applied Mathematics and Computation, vol. 167, no. 1, pp. 561-571, 2005.

[3] Y. Hu, Y. Luo, and Z. Lu, "Analytical solution of the linear fractional differential equation by Adomian decomposition method," Journal of Computational and Applied Mathematics, vol. 215, no. 1, pp. 220-229, 2008.

[4] I. Hashim, O. Abdulaziz, and S. Momani, "Homotopy analysis method for fractional IVPs," Communications in Nonlinear Science and Numerical Simulation, vol. 14, no. 3, pp. 674-684, 2009.

[5] A. K. Alomari, M. S. M. Noorani, R. Nazar, and C. P. Li, "Homotopy analysis method for solving fractional lorenz system," Communications in Nonlinear Science and Numerical Simulation, vol. 15, no. 7, pp. 1864-1872, 2010.

[6] M. Zurigat, S. Momani, Z. Odibat, and A. Alawneh, "The homotopy analysis method for handling systems of fractional differential equations," Applied Mathematical Modelling, vol. 34, no. 1, pp. 24-35, 2010.

[7] O. Abdulaziz, I. Hashim, and S. Momani, "Application of homotopy-perturbation method to fractional IVPs," Journal of Computational and Applied Mathematics, vol. 216, no. 2, pp. 574-584, 2008.

[8] O. Abdulaziz, I. Hashim, and S. Momani, "Solving systems of fractional differential equations by homotopy-perturbation method," Physics Letters A, vol. 372, no. 4, pp. 451-459, 2008.

[9] W. Guo-cheng, "A fractional variational iteration method for solving fractional nonlinear differential equations," Computers \& Mathematics with Applications, vol. 61, no. 8, pp. 2186-2190, 2011.

[10] S. Das, "Analytical solution of a fractional diffusion equation by variational iteration method," Computers \& Mathematics with Applications, vol. 57, no. 3, pp. 483-487, 2009. 
[11] Z. M. Odibat and N. T. Shawagfeh, "Generalized Taylor's formula," Applied Mathematics and Computation, vol. 186, no. 1, pp. 286-293, 2007.

[12] Z. Odibat, S. Momani, and V. S. Erturk, "Generalized differential transform method: application to differential equations of fractional order," Applied Mathematics and Computation, vol. 197, no. 2, pp. 467-477, 2008.

[13] V. S. Erturk and S. Momani, "Solving systems of fractional differential equations using differential transform method," Journal of Computational and Applied Mathematics, vol. 215, no. 1, pp. 142-151, 2008.

[14] G. Jumarie, "Modified Riemann-Liouville derivative and fractional Taylor series of nondifferentiable functions further results," Computers \& Mathematics with Applications, vol. 51, no. 9-10, pp. 1367-1376, 2006.

[15] G. Jumarie, "Table of some basic fractional calculus formulae derived from a modified Riemann-Liouville derivative for nondifferentiable functions," Applied Mathematics Letters, vol. 22, no. 3, pp. 378-385, 2009.

[16] N. Faraz, Y. Khan, H. Jafari, A. Yildirim, and M. Madani, "Fractional variational iteration method via modified RiemannLiouville derivative," Journal of King Saud University-Science, vol. 23, no. 4, pp. 413-417, 2011.

[17] B. Lu, "Backlund transformation of fractional Riccati equation and its applications to nonlinear fractional partial differential equations," Physics Letters A, vol. 376, no. 28-29, pp. 2045-2048, 2012.

[18] A. Arikoglu and I. Ozkol, "Solution of fractional differential equations by using differential transform method," Chaos, Solitons and Fractals, vol. 34, no. 5, pp. 1473-1481, 2007.

[19] S.-H. Chang and I.-L. Chang, "A new algorithm for calculating one-dimensional differential transform of nonlinear functions," Applied Mathematics and Computation, vol. 195, no. 2, pp. 799805, 2008. 


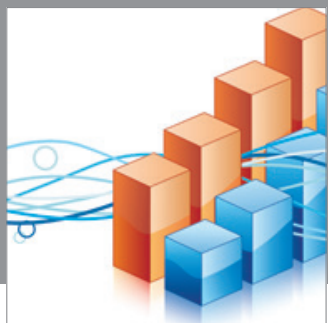

Advances in

Operations Research

mansans

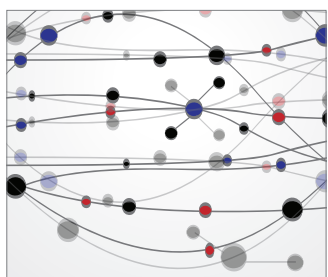

The Scientific World Journal
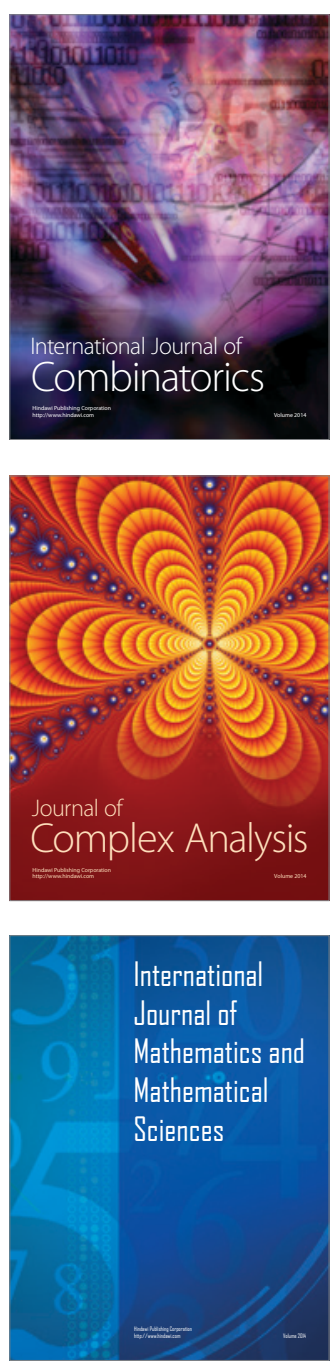
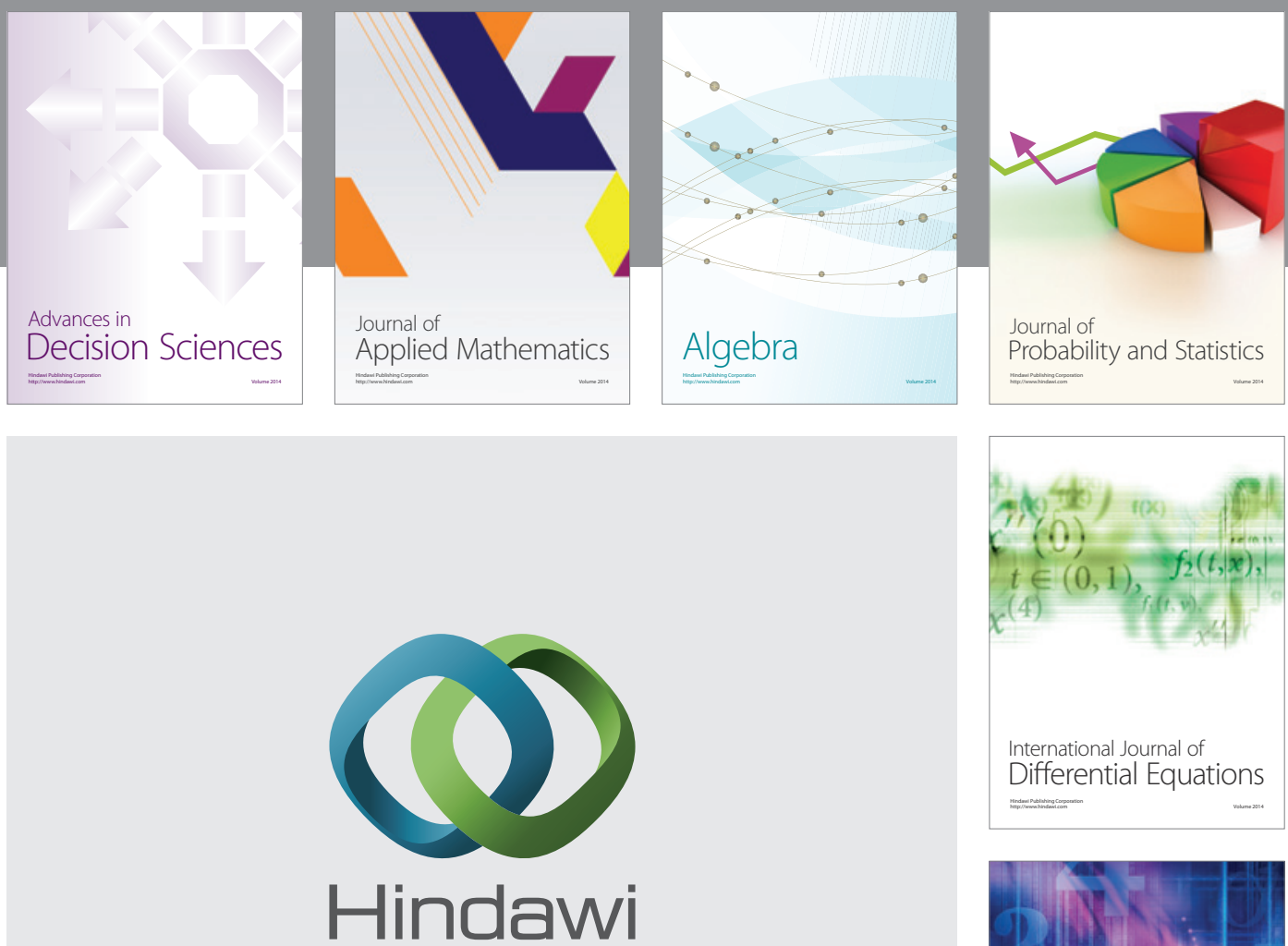

Submit your manuscripts at http://www.hindawi.com
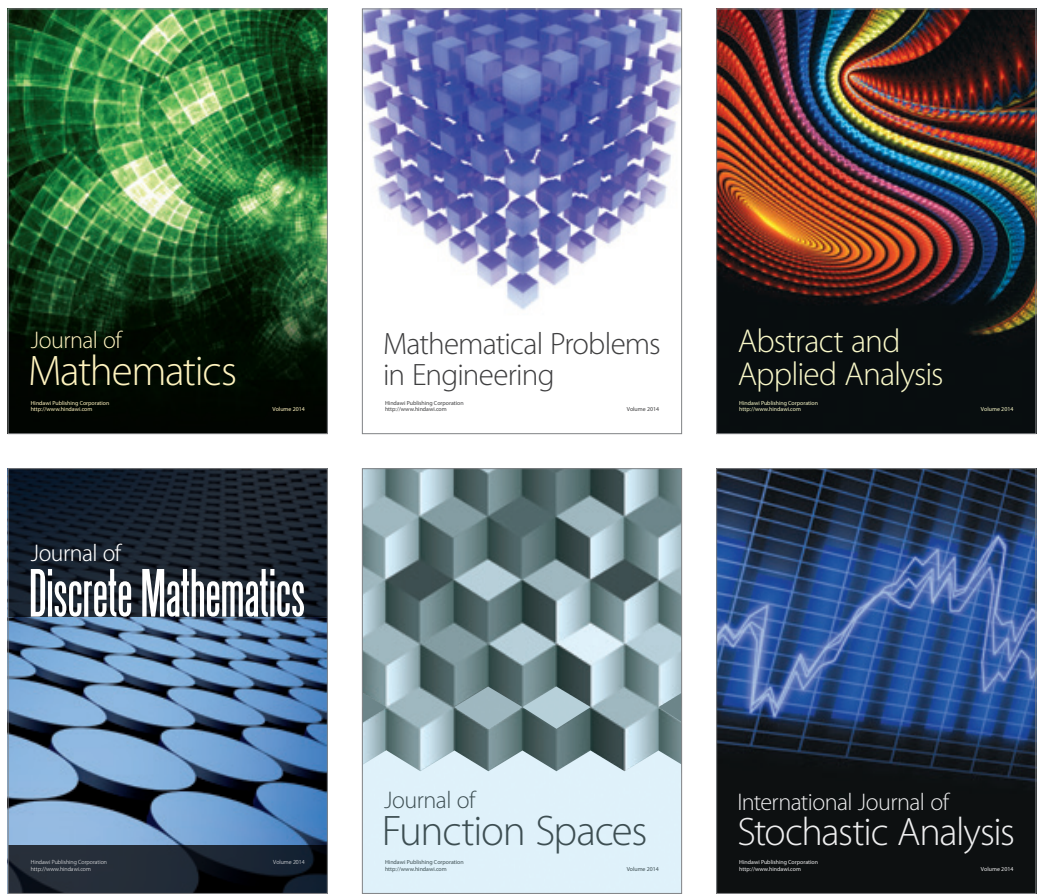

Journal of

Function Spaces

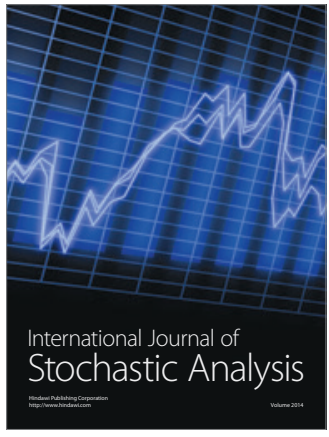

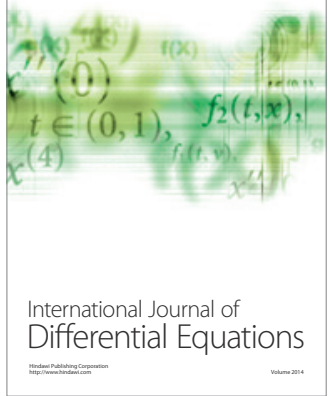
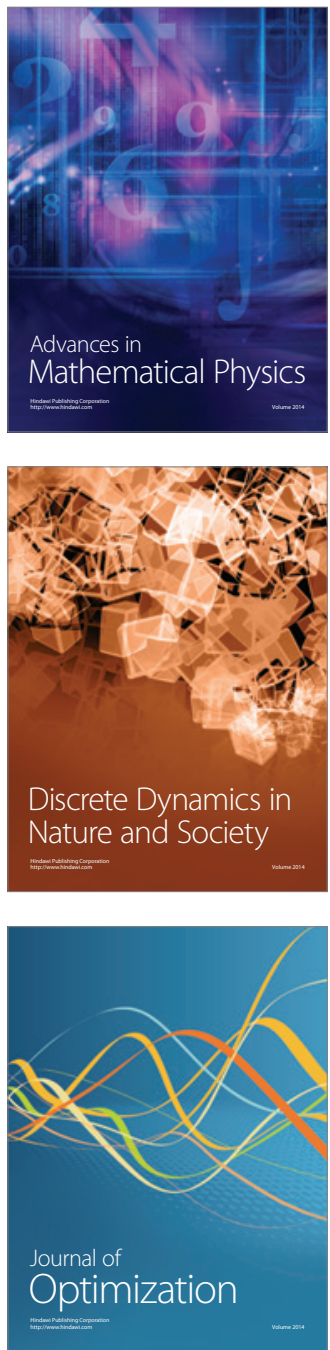